\title{
Structure, Function, and Cortical Representation of the Rat Submandibular Whisker Trident
}

\author{
Lydia Thé, ${ }^{1,2 \star}$ Michael L. Wallace, ${ }^{1,3 \star}$ Christopher H. Chen, ${ }^{1,4 \star}$ Edith Chorev, ${ }^{1,5 *}$ and Michael Brecht ${ }^{1,5,6}$ \\ ${ }^{1}$ Neural Systems and Behavior, Marine Biological Laboratory, Woods Hole, Massachusetts 02543, ${ }^{2}$ Department of Molecular and Cell Biology, University of \\ California, Berkeley, Berkeley, California 94720, ${ }^{3}$ Curriculum in Neurobiology, University of North Carolina, Chapel Hill, North Carolina 27599-7545, \\ ${ }^{4}$ Dominick P. Purpura Department of Neuroscience, Albert Einstein College of Medicine, Bronx, New York 10461, ${ }^{5}$ Bernstein Center for Computational \\ Neuroscience, Humboldt University of Berlin, 10115 Berlin, Germany, and ${ }^{6}$ Cluster of Excellence NeuroCure, Charité-Universitätsmedizin Berlin, 10117 \\ Berlin, Germany
}

Although the neurobiology of rodent facial whiskers has been studied intensively, little is known about sensing in other vibrissae. Here we describe the under-investigated submandibular "whisker trident" on the rat's chin. In this three-whisker array, a unique unpaired midline whisker is laterally flanked by two slightly shorter whiskers. All three whiskers point to the ground and are curved backwards. Unlike other whiskers, the trident is not located on an exposed body part. Trident vibrissae are not whisked and do not touch anything over long stretches of time. However, trident whiskers engage in sustained ground contact during head-down running while the animal is exploring or foraging. In biomechanical experiments, trident whiskers follow caudal ground movement more smoothly than facial whiskers. Remarkably, deflection angles decrease with increasing ground velocity. We identified one putative trident barrel in the left somatosensory cortex and two barrels in the right somatosensory cortex. The elongated putative trident-midline barrel is the longest and largest whisker barrel, suggesting that the midline trident whisker is of great functional significance. Cortical postsynaptic air-puff responses in the trident representation show much less temporal precision than facial whisker responses. Trident whiskers do not provide as much high-resolution information about object contacts as facial whiskers. Instead, our observations suggest an idiothetic function: their biomechanics allow trident whiskers to derive continuous measurements about ego motion from ground contacts. The midline position offers unique advantages in sensing heading direction in a laterally symmetric manner. The changes in trident deflection angle with velocity suggest that trident whiskers might function as a tactile speedometer.

\section{Introduction}

Rodent facial whiskers emerged as an important model system for sensory processing after the discovery of the cortical barrel field (Woolsey and Van der Loos, 1970). Since then, researchers have realized experimental advantages of the vibrissa/barrel cortex pathway, such as the ease of whisker manipulation (Van der Loos and Woolsey, 1973), highly controlled whisker stimulation (Simons, 1983), and combined in vivo and in vitro approaches (Finnerty et al., 1999). The power of mouse genetics also made

\footnotetext{
Received Oct. 9, 2012; revised Dec. 20, 2012; accepted Jan. 11, 2013.

Author contributions: L.T., M.L.W., C.H.C., E.C., and M.B. designed research; L.T., M.L.W., C.H.C., E.C., and M.B. performed research; L.T., M.L.W., C.H.C., E.C., and M.B. analyzed data; L.T., M.L.W., C.H.C., E.C., and M.B. wrote the paper.

This work was supported by the Marine Biological Laboratory, the National Institute of Mental Health (Training Grant 5R25MH059472), Humboldt Universität zu Berlin, the Bernstein (enter for Computational Neuroscience Berlin, the German Federal Ministry of Education and Research (Förderkennzeichen 01GQ1001A), the Deutsche Forschungsgemeinschaft (EXC 257, Neurocure), and the European Research Council (grant to M.B.). M.L.W. was supported by a National Research Service Award from National Institute of Neurological Disorders and Stroke (1F31NS077847). We thank Brigitte Geue, Christian Ebbesen, Luigs \& Neumann, Heka, and the Neural Systems and Behavior staff.

*L.T., M.L.W., C.H.C., and E.C. contributed equally to this work.

The authors declare no competing financial interests.

Correspondence should be addressed to Michael Brecht, Neural Systems and Behavior, Marine Biological Laboratory, 7 MBL Street, Woods Hole, MA 02543. E-mail: michael.brecht@bccn-berlin.de.

DOI:10.1523/JNEUROSCI.4770-12.2013

Copyright $\odot 2013$ the authors $\quad 0270-6474 / 13 / 334815-10 \$ 15.00 / 0$
}

the large and accessible whisker representations a focus of interest for novel optic and optogenetic techniques (O'Connor et al., 2009; Scanziani and Häusser, 2009).

Controlled whisker deflection enabled investigators to describe receptive fields in the vibrissa-barrel cortex pathway in great detail for both spiking (Simons, 1978, 1985) and postsynaptic responses (Moore and Nelson, 1998; Zhu and Connors, 1999; Brecht and Sakmann, 2002; Brecht et al., 2003; Manns et al., 2004). After an initial focus on anesthetized animals, research on the vibrissal system has broadened to also include investigations in awake, behaving animals (Carvell and Simons, 1990; von Heimendahl et al., 2007) and the analysis of active touch (Fee et al., 1997; Crochet and Petersen, 2006; Deschênes et al., 2012). Nevertheless, our knowledge of whisker-mediated behaviors lags behind the advanced state of anatomical and physiological analysis of the vibrissal system (Brecht, 2007). In recent years, interest in vibrissa biomechanics has increased (Hartmann et al., 2003; Neimark et al., 2003). "Artificial whisking" preparations have been used to characterize cortical responses under more active touch-like conditions (Derdikman et al., 2006). Evidence has been provided that vibrissa micromotions during surface contacts form "kinetic signatures" encoding surface properties (Arabzadeh et al., 2005). It has also been argued that specific kinetic events referred to as "stick and slip" movements underlie vibrissal texture discrimination (Ritt et al., 2008; Jadhav and Feldman, 2010). 
The thousands of studies on the macrovibrissae should not blind one to the fact that most of the somatosensory system remains unexplored. Most of the $\sim 300$ whiskers on a rat are organized in arrays (e.g., the upper and lower lip microvibrissae, and the paw whiskers) that we know little about. The few studies on microvibrissae immediately suggested functional differences between macro- and microvibrissae at the behavioral level (Brecht et al., 1997; Anjum et al., 2006) and at the level of cortical representation (Elston et al., 1997). Elucidating the functional diversity of whisker signaling holds promise for a generalized understanding of whisker sensing. Here we compared the characteristics of the largely unstudied submandibular whisker trident with those of facial whiskers. Specifically we investigated: (1) what is the morphology of the trident whisker array?; (2) how are they used in behavior?; (3) which biomechanical factors shape their contacts?; (4) how are these whiskers represented in the cortex?; and (5) how do cortical neurons representing trident whiskers respond to sensory stimulation?

The whisker trident morphology, behavior, and response characteristics contradict the idea that these whiskers acquire high-resolution tactile information. Instead, biomechanical and behavioral analyses suggest that trident whiskers provide information about ego motion. We discuss the hypothesis that the whisker trident functions in heading-direction estimation and as a tactile speedometer.

\section{Materials and Methods}

All experiments complied with German and American regulations on animal welfare and were approved by ethics committees in Berlin, Germany, and Woods Hole, MA, respectively.

Morphology. Long-Evans hooded rats of either sex $(n=18)$, Wistar rats of either sex $(n=5)$, and C57BL mice $(n=3)$ of either sex were used in the morphological analysis. Animals were killed with a $0.4 \mathrm{ml}$ injection of pentobarbital or an overdose inhalation of isoflurane. The trident whiskers were photographed using an AxioCamMRc5 camera (Zeiss) mounted on a Stemi stereomicroscope (Zeiss) and connected to a computer system for digital image acquisition. An incision in the submandibular region was made parallel to the trident whiskers and the subcutaneous fat and connective tissue surrounding the capsule were removed to expose follicles.

Behavior. We investigated trident whisker use in a range of behavioral settings, specifically, in the home cage, in a novel behavioral arena, and in open fields. We also had rats balance over narrow beams (broomsticks) and observed animals running in narrow slits and tunnels. To observe foraging, we trained animals to collect chocolate pellets in an open field setting. In all of these settings, we observed animals by eye, videotaping, high-speed videography, and photography. Much of the filming was done in a Plexiglas chamber. Rats were housed with a $12 \mathrm{~h} \mathrm{light/dark} \mathrm{cycle}$ at $21^{\circ} \mathrm{C}$.

Biomechanics. To make biomechanical measurements of the trident and facial whiskers, we positioned an anesthetized rat head posted to a manual micromanipulator over a bicycle tire $(69 \mathrm{~cm}$ outer diameter) so that the midline trident whisker or a facial whisker was in contact with the tire and so that the tire surface was approximately parallel to the ventral neck fur of the rat.

Videography. We recorded whisker movements at 240 frames/s using a high-speed video camera (S9100; Nikon) and a Basler camera. Ground velocity and direction were controlled by rotating the tire at different speeds and directions. Ground velocity was calculated post hoc. Whisker angle measurements were made frame by frame using ImageJ software (http://rsb.info.nih.gov/ij/), where $0^{\circ}$ was approximately perpendicular to the ventral neck fur and negative angles corresponded to the angles where the tip of the whisker was more posterior.

Electrophysiology. We used standard physiological techniques described in detail in Margrie et al. (2002) and Brecht and Sakmann (2002) for whole-cell recordings. Extracellular recording and hand mapping of receptive fields were performed in four experiments (one in the right hemisphere and three in the left hemisphere) with a (1 M $\Omega$ ) tungsten electrode. Signals were amplified, differentially filtered for spikes, and sent to an audiomonitor using an NPI extracellular amplifier.

Animal preparation. Long-Evans rats (P19-P25, $n=14)$ were anesthetized using urethane $(1.4 \mathrm{~g} / \mathrm{kg}$, i.p.). Incised tissue was locally anesthetized with lidocaine. A rectal probe monitored body temperature and a homeothermic blanket (FHC) maintained it at $37 \pm 0.5^{\circ} \mathrm{C}$. For facial whisker barrel experiments, a craniotomy was made above the somatosensory cortex ( $2.5 \mathrm{~mm}$ posterior to and $5.5 \mathrm{~mm}$ lateral to bregma). For submandibular-trident whisker barrel experiments, a craniotomy was made $0.5 \mathrm{~mm}$ anterior to and $5 \mathrm{~mm}$ lateral to bregma. Electrodes were arranged to enter perpendicular to the cortex. Recording depths ranged from 248 to $1342 \mu \mathrm{m}$ with mean of $560 \pm 280 \mathrm{SD}$. Eighty-three percent of our cells were recorded at a depth of $<700 \mu \mathrm{m}$ and were most likely layer $2 / 3$ regular spiking neurons.

Whisker stimulation. Puffs of air were presented to either facial or submandibular trident whiskers every $5 \mathrm{~s}$ for at least 10 trials. Air puffs persisted for $1 \mathrm{~s}$. Care was taken such that only the experimental whiskers were deflected by the air puff. Air puffs were generated from pulses of compressed air, delivered by a computer triggered airflow controller (Sigmann Elektronik) and were applied through a stiff micropipettor tip with a $2 \mathrm{~mm}$ opening positioned $10-15 \mathrm{~mm}$ rostrolaterally from the whiskers. The air-puff stimulus was moved across the whisker array and 20 air-puff stimuli were applied at the most effective positions.

Whole-cell recordings. Pipettes were pulled to 3-8 M $\Omega$ (P1000, Sutter Instruments) from filamented $(0.25 \mathrm{~mm})$ borosilicate glass (outer diameter $2.0 \mathrm{~mm}$, inner diameter $1.5 \mathrm{~mm}$; Hilgenberg). Intracellular solutions were composed of the following (in mM): K-gluconate 130, Na-gluconate 10, HEPES 10, phosphocreatine 10, MgATP 4, GTP 0.3, $\mathrm{NaCl} 4$, and biocytin $0.3-1 \%$ at $\mathrm{pH}$ 7.2. Signals were amplified (Cornerstone amplifier; Dagan), filtered at $3-10 \mathrm{kHz}$, and digitized at $20 \mathrm{kHz}$ (ITC-16; Instrutech) using HEKA software. Records were exported and analyzed in MATLAB version 2012a (Natick).

Analysis. PSP amplitudes for each cell were calculated from the average trace of at least 10 trials. To determine the EPSP amplitude, the resting membrane potential was subtracted from the peak of the initial depolarization during the air pulse. The EPSP amplitude was then used to find the $20 \%$ and $80 \%$ depolarization values to compute the rise time. Because all traces had IPSPs after the initial EPSP, the IPSP magnitude was calculated by subtracting the subsequent negative deflection from the peak of the EPSP. The timing of each peak was used to calculate the PSP latencies. Note that these are working definitions of IPSPs and EPSPs based on their hyperpolarizing or depolarizing membrane potential trajectories and not pharmacologically isolated potentials. It is almost certain that both IPSPs and EPSPs come about by a mixture of inhibitory and excitatory inputs with a dominating inhibitory or excitatory contribution, respectively.

Histochemical visualization of barrel patterns. Animals were deeply anesthetized and perfused transcardially with prefix, followed by $4 \%$ paraformaldehyde (PFA). Brains were removed, hemispheres were separated, and cortices were flattened between two glass slides separated by clay spacers. Glass slides were weighed down with small ceramic weights for $\sim 3 \mathrm{~h}$. Afterward, flattened cortices were stored overnight in 2\% PFA and $80 \mu \mathrm{m}$ sections were cut on a Vibratome. Sections were stained for cytochrome-oxidase activity using the protocol of Wong-Riley (1979). Subsequently, barrel shapes were drawn with Neurolucida software (Microbrightfield) using a Zeiss Axioplan microscope fitted with $10 \times$ and $2 \times$ objectives.

\section{Results \\ Morphology of rat trident whiskers}

We characterized the submandibular trident whiskers and compared them with the well studied facial whiskers. The submandibular whisker trident consists of three whiskers on the animal's chin: a midline and a pair of mirror-symmetric left and right trident whiskers (Fig. 1A). The origins of the three whiskers form a line along the mediolateral body axis. We analyzed the gross 

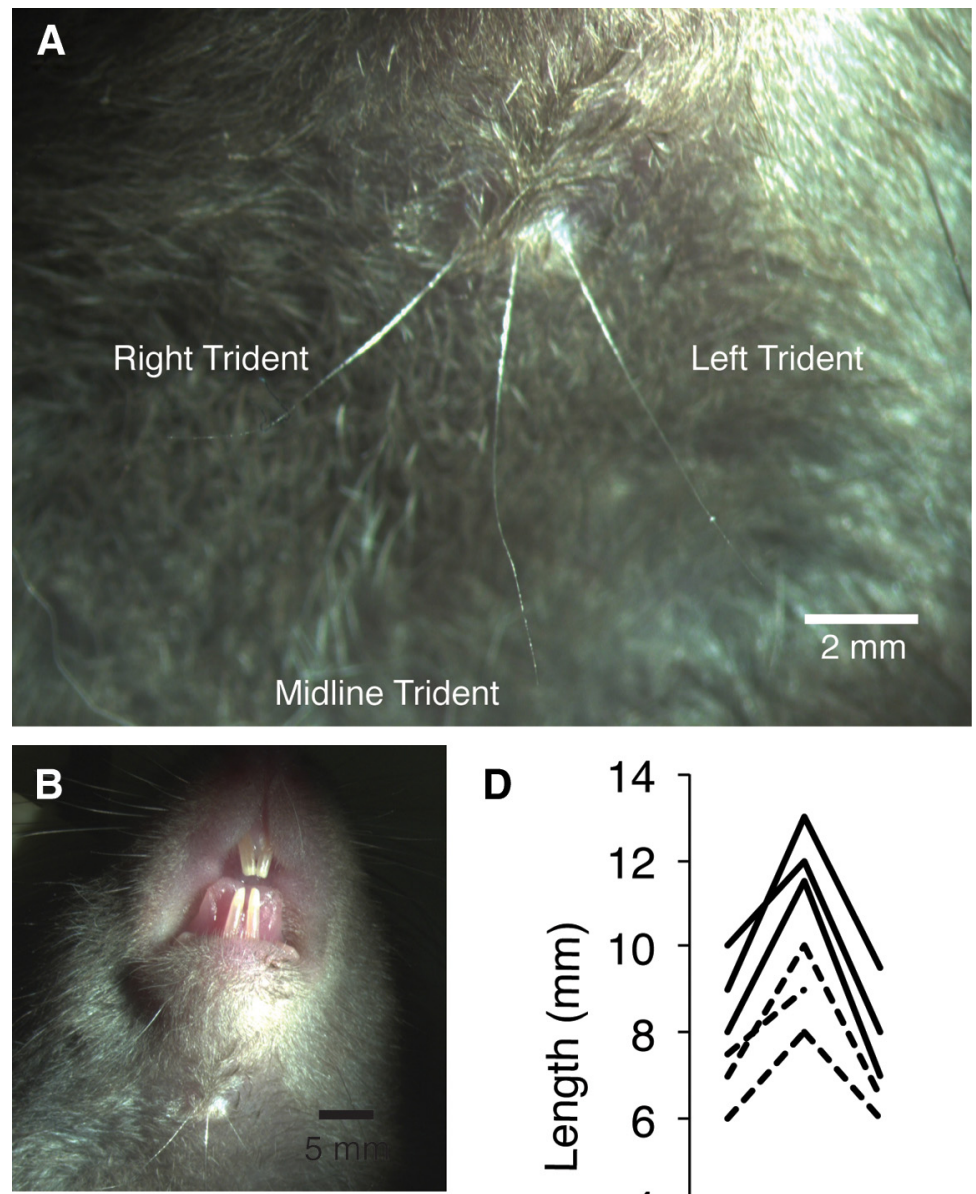

D
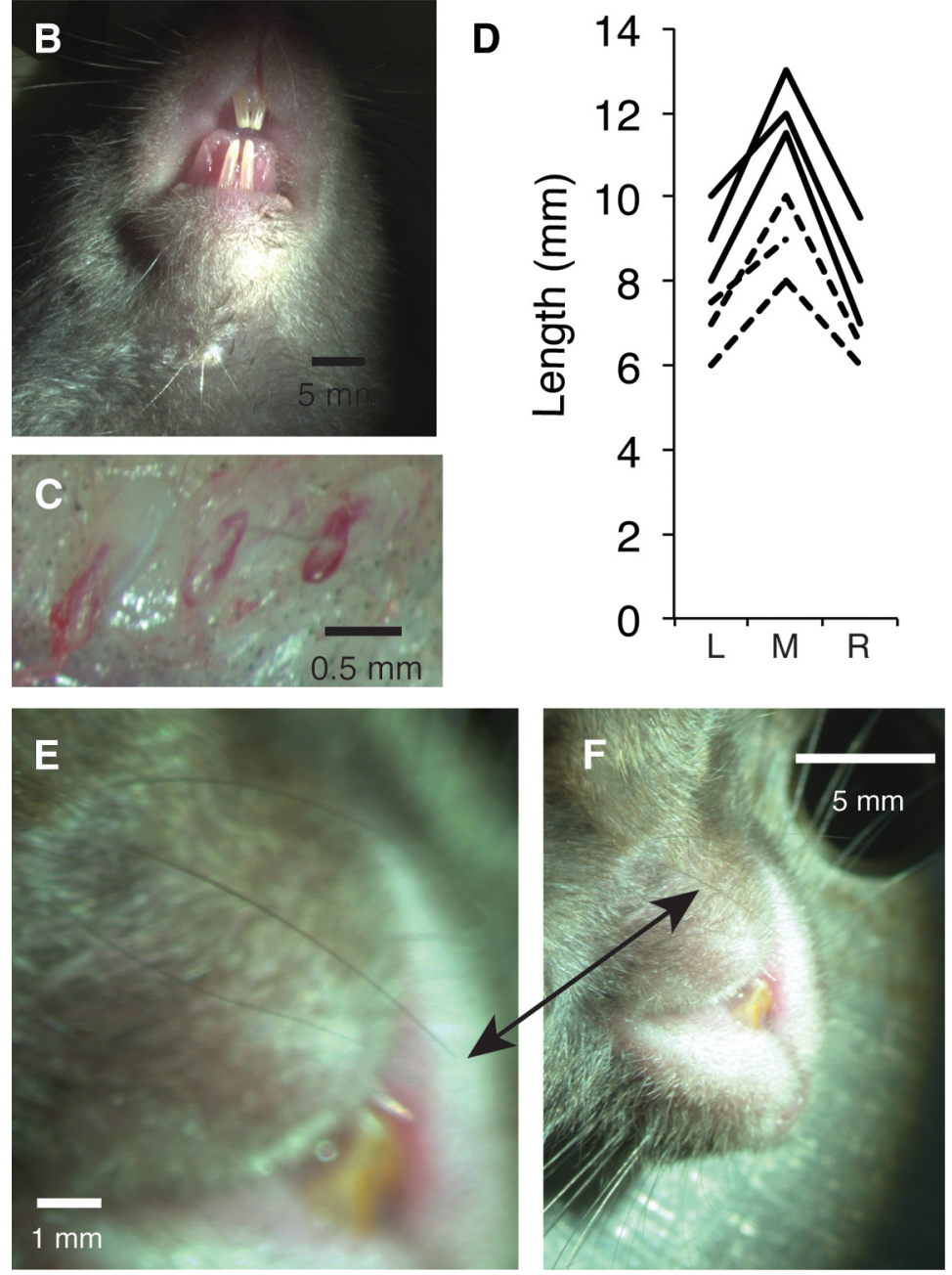

Figure 1. Anatomy of rat and mouse trident vibrissae. $\boldsymbol{A}$, Right, middle, and left trident whiskers of the black and white hooded rat. Scale bar, $2 \mathrm{~mm}$. $\boldsymbol{B}$, Larger view of rat trident whiskers demonstrating their location in the submandibular region. Scale bar, 5 $\mathrm{mm}$. C, Each trident whisker follicle is encapsulated by a blood sinus. Scale bar, $0.5 \mathrm{~mm}$. D, Length of the left (L), middle (M), and right (R) trident whiskers in 6 rats. Continuous lines refer to adult animals; dashed lines refer to 3- to 5-week-old animals. $\boldsymbol{E}$, Three trident whiskers in a C57BL mouse. Scale bar, $1 \mathrm{~mm}$. F, Larger view of mouse trident whiskers demonstrating their location in the submandibular region. Scale bar, $5 \mathrm{~mm}$. morphology of rat trident whiskers in 23 rats, all of which had the midline trident whisker and 5 of which were missing either the left or right trident whisker. All three trident whiskers point to the ground and are curved backwards. We inspected all of the $\sim 300(2 \times \sim 150)$ whiskers or putative sinus hairs of the rats, but we did not detect any other whisker that was systematically (across animals) unpaired with respect to hemibodies. We conclude that the unpaired arrangement of the midline trident whisker is a unique feature of the trident system.

The trident whiskers are spatially separated from other whiskers on the body surface and-unlike most other whiskers-are not in an exposed position that would favor contact with objects or obstacles (Fig. 1B).

Dissection of whisker follicles revealed that trident whiskers are proper sinus hairs with follicles encapsulated by a blood sinus (Fig. 1C). In dissections of three animals with three trident whiskers, we observed exactly 3 blood sinuses in the submandibular region. In one rat that had only two trident whiskers, we also observed only two whisker follicles with blood sinuses, suggesting that the absent whisker came about by a true polymorphism rather than by a transient whisker loss. The middle whisker had the longest length $(10.9 \pm 1.3 \mathrm{~mm} \mathrm{SD}, p<0.0001)$, whereas the left and right whiskers were near equal in length (right, $7.7 \pm 1.2 \mathrm{~mm}$ SD; left, $8.4 \pm 1.2 \mathrm{~mm}$ SD; Fig. 1D).

The trident whiskers had a similar position and morphology in C57BL mice (Fig. 1E,F). The conserved morphology may suggest that sustained selection pressures maintain the architecture of the trident whisker arrangement.

\section{Trident whisker use in behavior}

Trident contacts are rare

Trident whisker use was investigated in a variety of behavioral settings. Trident whiskers were, over long stretches of time, not in contact with objects or obstacles. This was particularly true for home-cage settings and arenas familiar to the animal. Tasks such as broomstick balancing and tunnel running occasionally, but not consistently, led to trident whisker contact.

Engagement of trident and facial whiskers in head-down running

The most consistent contact patterns of trident whiskers were ground contacts during head-down running (Fig. $2 A, B$ ). Head-down running is a body posture tied to locomotion and involves down- 

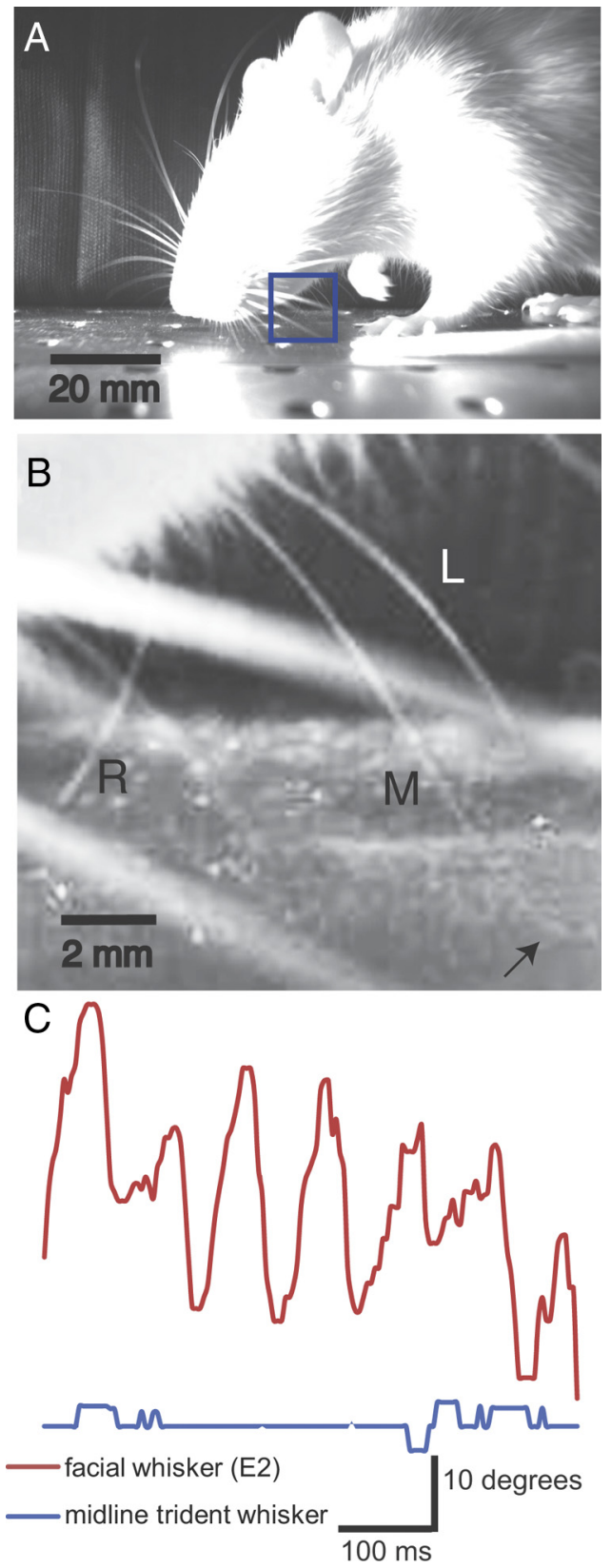

Figure 2. Behavior: ground contact of trident vibrissae during head-down running and absence of whisking. $A$, Still image from a high-speed infrared video of a rat running head down in an environment novel to the animal. The video was taken under bright infrared illumination but under low levels of ambient visible light. Note the position of the paw, which indicates that the frame was taken at the middle of the animal's stepping cycle. $\boldsymbol{B}$, Enlargement of the trident whisker region (outlined with the blue box in $A$ ). Brightness and contrast have been enhanced by image processing to increase the visibility of whiskers. The left (L), middle $(M)$, and right $(R)$ trident whiskers are visible. Inspection of the video sequence shows that the left and right trident whiskers are free in air, whereas the midline trident is dragging on the ground (arrow). C, Rat E2 facial (red) and midline trident (blue) whisker deflection angles during $500 \mathrm{~ms}$ recording. Recordings were collected using high-speed videography (240 frames/s) taking the trident whisker tip and the whisker origin as references for angular measurements. Facial whiskers demonstrated large deflections $\left(30^{\circ}\right)$, whereas trident whiskers showed only small and no rhythmic movements.

ward bending of the head by $\sim 45^{\circ}\left(43^{\circ} \pm 6^{\circ}\right.$, data from 4 animals in 7 high-speed videography sessions). Rats engage in head-down running during their initial exploration of novel environments. During the later stages of exploration, animals often raise their heads, rear on their hindpaws, or run without head-ground con- tact. Head-down running was reliably observed the first time an animal encountered an enclosure. However, habituation was very fast: by the second time an animal was exposed to a novel environment, the fraction of time the animal spent head-down running was greatly reduced.

Head-down running is also observed in known environments, although only when animals are foraging for food. The repositioning of the head brings the nose close to the floor, leading to intermittent contact of facial whiskers with the ground, but sustained trident ground contact. In the head-down posture, the base of the trident whiskers are on average $5.5 \pm 1.5 \mathrm{~mm}$ above ground, bringing the floor just within range of the shorter left and right trident whiskers and well within reach of the midline trident. Consistent with this, we observed the midline whisker to be in sustained ground contact during head-down running. As the head bobbed up and down through the animal's stepping cycle, the strength of ground contact and the bending of the midline whisker varied slightly. The extent to which the left and right trident whisker contacted the ground also varied during headdown running. In some cases, they engaged in sustained contact and in others, we observed them free in the air (Fig. 2C). In addition to ground contacts, we often observed wall contact of trident whiskers when animals reared in apposition to a wall.

\section{Trident whiskers do not whisk}

Rats and mice rhythmically palpate their facial whiskers in a movement known as whisking. We investigated whether trident whiskers are also motile. We compared the E2 facial whisker with the midline trident whisker using high-speed videography (Fig. $2 C)$. We found that the facial whiskers demonstrated characteristic rhythmic sweeping deflections of up to $30^{\circ}$. We also observed that head-down running was associated with intense whisking of the facial macrovibrissae. These whisker positions and whisking amplitudes demonstrate that a fraction of facial macrovibrissae gently and intermittently touch the ground during head-down running. Trident whiskers, in contrast, display only small movements and no rhythmic movements. These data suggest that the trident whiskers may play a distinct sensory role from facial whiskers and suggest that there may be differences in the way information from the trident whiskers is processed.

\section{Trident whisker biomechanics during ground motion}

After our behavioral observations of the trident whisker engaged during head-down running, we designed experiments to study the biomechanics of trident whisker contact and deflection during ground movement. We placed the midline trident whisker of an anesthetized rat with a head-down tilt above a large tire that enabled near planar displacement of the ground. We used highspeed videography to record midline trident whisker motions and angular deflection patterns during backward and forward ground movement (Fig. 3A). Interestingly, the whisker deflection pattern was distinct in forward and backward ground movement. The trident whisker had a smooth deflection pattern when the ground was moving backward, but a jerky deflection pattern when the ground was moving forward (Fig. 3A). This difference was quantified by calculating the root mean square of the whisker deflection angle (Fig. 3C). The smooth whisker movement during backward ground movement seems to result from a stable balance between frictional forces from ground movement and the forces pressing the trident whisker on the ground. This smooth whisker movement is in contrast to forward movement, which charges up the backwards-curved whisker like a spring, resulting in abrupt discharges of the spring force and jerky move- 
A
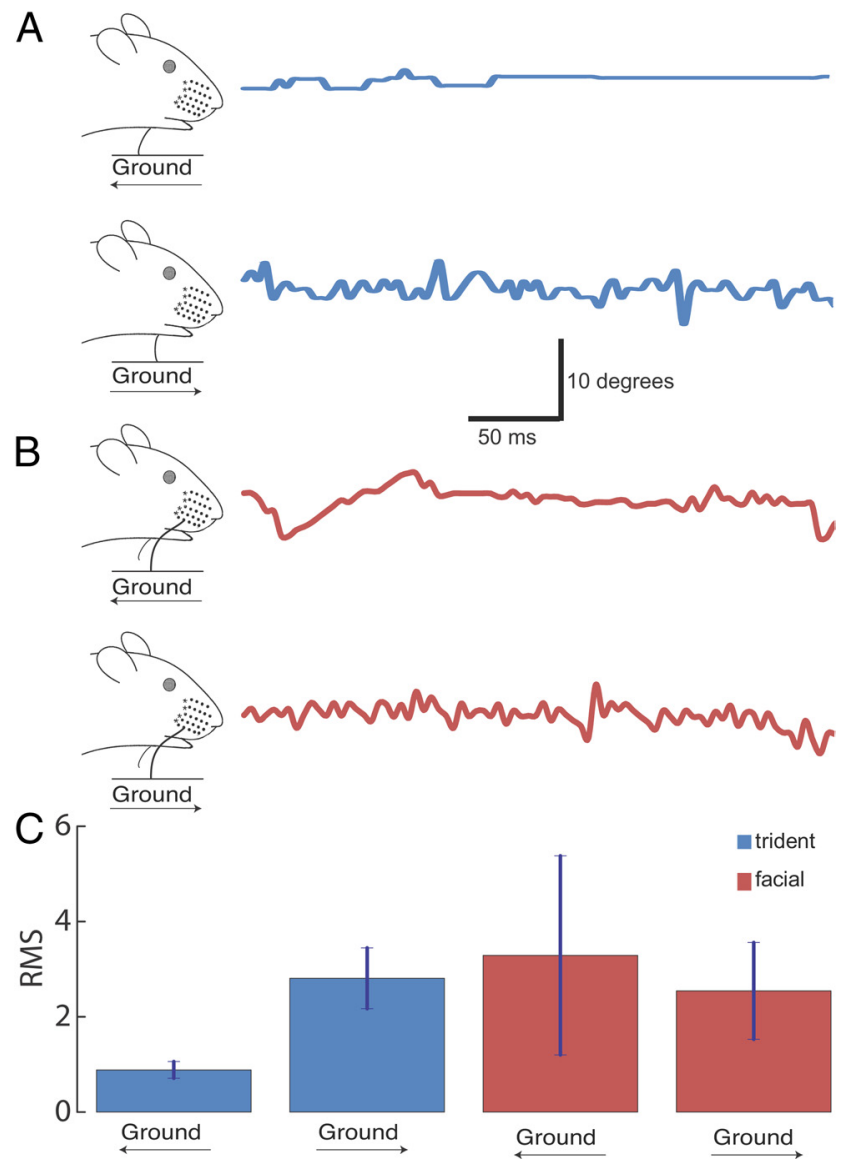

Figure 3. Backwards ground movement (corresponding to forward movement of the animal) yields smooth deflections of trident but not of facial whiskers. $A$, Left, Illustration depicting direction of ground movement while the midline trident whisker was in contact with the ground during the recording period. $\boldsymbol{A}$, Right, Blue line indicates trident whisker deflection angle over the course of a trial. Data were collected using high-speed videography ( $240 \mathrm{frames} / \mathrm{s}$ ) taking the trident whisker tip and the whisker origin as references for angular measurements. Note the smooth and jerky deflection pattern for backward and forward ground movement, respectively. $\boldsymbol{B}$, Left, Illustration depicting the direction of ground movement while the facial whisker was in contact with the ground during the recording period. $\boldsymbol{B}$, Right, Red line indicates facial whisker deflection angle over the course of a trial. C, Average root mean squared values of trident (blue) and facial (red) whisker deflection angles during a $500 \mathrm{~ms}$ trial ( $n=2$ animals). Surprisingly, different ground velocities (range 1-50 cm/s) resulted in different deflection patterns at our sampling rate $(240 \mathrm{~Hz})$ and data were pooled across velocities. Animal averages were calculated from 3 different ground velocities per animal. Bars indicate mean \pm SE.

ments. In contrast to the trident whisker, the facial whisker did not respond differently to forward versus backward ground movement (Fig. 3B,C) and overall followed the ground much less smoothly. These data suggest that the trident whiskers have a unique ability to follow ground movement smoothly and are sensitive to ground motion direction, whereas facial whiskers are not.

Given the sensitivity of the trident whisker to the direction of ground movement, we hypothesized that the whiskers may also be sensitive to changes in velocity. To test this, we recorded whisker angles during backward ground movement (Fig. 4A-D). For both low (Fig. 4A) and high (Fig. 4B) speeds, the whisker was most deflected (more negative deflection angles) at low velocities and less deflected at higher ground velocities. This surprising result was seen in all videos $(n=7)$ in which we accelerated the ground below the animal. This suggests, counterintuitively, that at slower velocities, larger frictional forces from the ground result in larger whisker deflections (Fig. 4C,D) than at faster velocities. These findings suggest a dominating contribution of static friction (which decreases with speed) over dynamic friction (which increases with speed) in the trident-ground interaction. Our data show that the trident whisker is sensitive to both direction and velocity of ground movement.

\section{Identification of putative trident barrels}

Given the unique morphology of the trident whiskers, we next searched for the putative barrels corresponding to the trident whiskers in the rat somatosensory cortex. We prepared flattened somatosensory cortices of both cortical hemispheres of rats $(n=$ 5 ). We stained these cortices for cytochrome-oxidase activity and visualized cortical patterns in both hemispheres (Fig. 5A). We then drew barrel patterns corresponding to the rat's body surface through serial sections (Fig. $5 B$ ). Having reconstructed the barrel pattern in both hemispheres, we used five complementary search criteria that uniquely identified putative trident whisker barrels:

\section{Coordinates}

We used coordinates from our physiological experiments and retranslated these coordinates to body-barrel map locations using the map of Chapin and Lin (1984). Trident responses were observed in 5 of 6 physiological experiments between lateral 5 $\mathrm{mm}$ (L5)/anterior $0 \mathrm{~mm}$ (A0) and at L5/A0.5. To search the barrel maps at these coordinates, we used the conspicuous medial end of barrel row $\mathrm{G}$ and $\mathrm{H}$ as a landmark, which is situated at L5.5/ A0.5 mm in the map of Chapin and Lin (1984). In four of the five right hemispheres, the L5/A0 to L5/A0.5 coordinates overlapped partially or fully with one large barrel shown in dark blue in Figure $5 B-D$ (the putative midline trident barrel). In two of the five left hemispheres, these coordinates overlapped partially or fully with a medium-sized isolated barrel shown in light blue on the right side of Figure $5 B-D$ (the putative right trident barrel). In one of the five left hemispheres, these coordinates did not overlap with any barrel, but were equidistant to the medium-sized isolated barrel shown in light blue in Figure $5 B-D$ (the putative right trident barrel) and a small putative lower jaw barrel. In the remaining three cases, these coordinates overlapped with two different small putative lower jaw barrels and one did not overlap with any barrel.

\section{Extracellular mapping}

In four experiments (one in the right hemisphere and three in the left hemisphere), we combined extracellular recordings and hand mapping of receptive fields with lesions and histological reconstruction of barrel patterns. In these mapping experiments, trident responses were consistently observed in a strip of recording sites in between laterally located responses to facial whisker deflections and medially located responses to the forepaw stimulation. Therefore, our mapping experiments supported the barrel assignment illustrated in Figure 5.

\section{Topography}

The isolated topographic location of trident whiskers in the face (Fig. $1 B$ ) allowed us to identify one isolated barrel posterior from the more closely spaced lower jaw whisker barrels in the left hemisphere (Fig. 5B-D) and two isolated barrels posterior from the more closely spaced lower jaw whisker barrels in the right hemisphere (Fig. 5B-D, putative midline and left trident barrels).

\section{Whisker size and barrel size}

The trident whiskers are more prominent and longer than the more frontal lower jaw whiskers. Therefore, we reasoned that trident barrels should be somewhat larger than lower jaw barrels, and this was indeed the case for the putative trident barrels at the 
correct coordinates and the appropriate topographic position (Fig. $5 B-D$ ).

\section{Number}

We reasoned that there should be three putative trident barrels for the three trident whiskers. We verified the presence of all three trident whiskers before perfusing animals for histology and identified in all five brains three putative trident barrels at the correct coordinates and appropriate topographic position (Fig. 5B-D).

\section{Characteristics of putative trident barrels}

Hemispheric asymmetry and ghost zone Perhaps the most conspicuous feature of the putative cortical trident representation is the striking hemispheric asymmetry of putative trident barrels that are visible even in whole mounts of the rat somatosensory cortices (Fig. 5A,B). This asymmetry arises not only because the right hemisphere contains two of the three putative trident barrels, but also because the putative midline trident barrel is very large. In the left hemisphere between the lower jaw barrels and the putative right trident barrel, we consistently observed a dark (cytochrome-oxidase active) zone without sharp borders. This "ghost zone" is at the mirror-symmetric position of the right midline trident barrel. From our extracellular recording and receptive fieldmapping experiments, it appeared that sites directly adjacent to or in the ghost zone respond to the right and midline trident whisker.

Putative trident barrels are isolated Most whiskers appear in groups on the body and are represented in densely packed barrel clusters in the somatosensory cortex. Even whiskers with somewhat isolated positions on the head, such as the supraorbital whisker or the whisker associated with the pinna, are represented in barrels directly adjacent to the mystacial barrels (Fig. 5B, top left two barrels). Putative trident barrels, however, do not follow this compactrepresentation rule and map to isolated positions in the cortical representation (Fig. 5B-D).

\section{Size of putative trident barrels}

The putative midline trident barrel was the largest whisker barrel identified in the rat brain (Fig. $5 E$ ); however, the difference between E2, the largest facial barrel, and the putative midline trident barrel was not significant. The putative midline trident barrel was approximately twofold larger than the putative left trident barrel or the C3 barrel (an average-sized facial barrel) and threefold larger than the putative right trident barrel. All of these differences were highly significant $(p<0.001, t$ test). The midline trident whisker was on average $\sim 11 \mathrm{~mm}$ long and the right and left trident whiskers were $\sim 8 \mathrm{~mm}$ long. Given that whisker length is approximately correlated with barrel area for facial whiskers, the large size of the putative midline trident barrel and the

\section{B Fast Ground Movement}

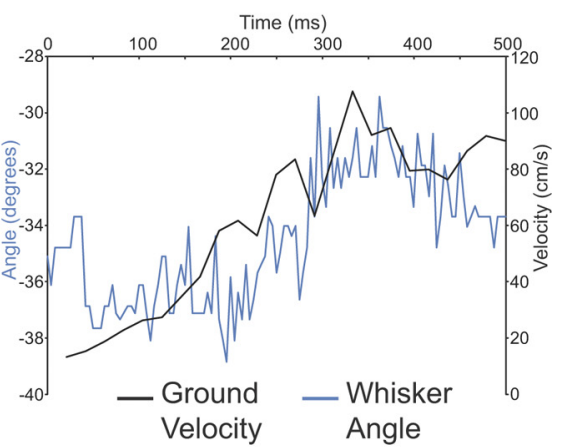

D Midline trident deflection at high and low speed

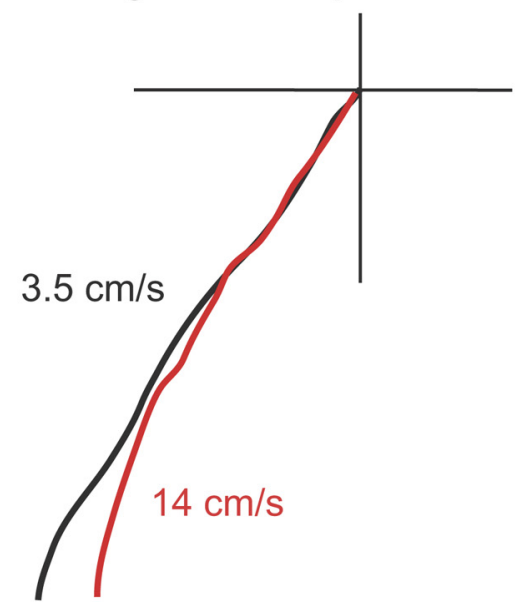

Figure 4. Changes in trident whisker angle during ground acceleration. $A, B$, Representative trials (for slow and fast backward denominatation: $0^{\circ}$ was a line drawn perpendicular to the rat's fur. More negative angles correspond to more strongly deflected whisker positions; less negative angles correspond to smaller deflections. C, Sample frame from a high-speed video taken during ground movement with respect to the origin (location where the trident whisker meets the fur), demonstrating that trident whisker defects more at slower ground velocity (black trace).

marked size difference between the putative midline barrel and right and left trident barrels comes as a surprise.

\section{Shape of putative trident barrels}

The putative trident midline barrel was greatly elongated along the anterior-posterior body axis (Fig. 5B-D). We analyzed barrel shapes by quantifying maximal and minimal Feret diameters, which represent the longest and shortest dimension of the barrel outline independent of its angular rotation, respectively. Remarkably, the putative midline barrel with a maximal Feret diameter of approximately $1.1 \mathrm{~mm}$ was the longest whisker barrel in the rat brain by a wide margin (Fig. $5 F$ ). At the same time, the average minimum Feret diameter was only $0.3 \mathrm{~mm}$, significantly less than that of the facial E2 or C3 whisker. These differences reflect the more compact shape of facial barrels.

Poorly defined barrel borders

Most whisker barrels in the rat cortex are sharply delineated, but this was not the case for the putative trident barrels.

\section{Laminar organization}

L4 was much thinner in trident barrels (100-200 $\mu \mathrm{m}$ thickness) than in whisker barrels (200-300 $\mu \mathrm{m}$ thickness). 


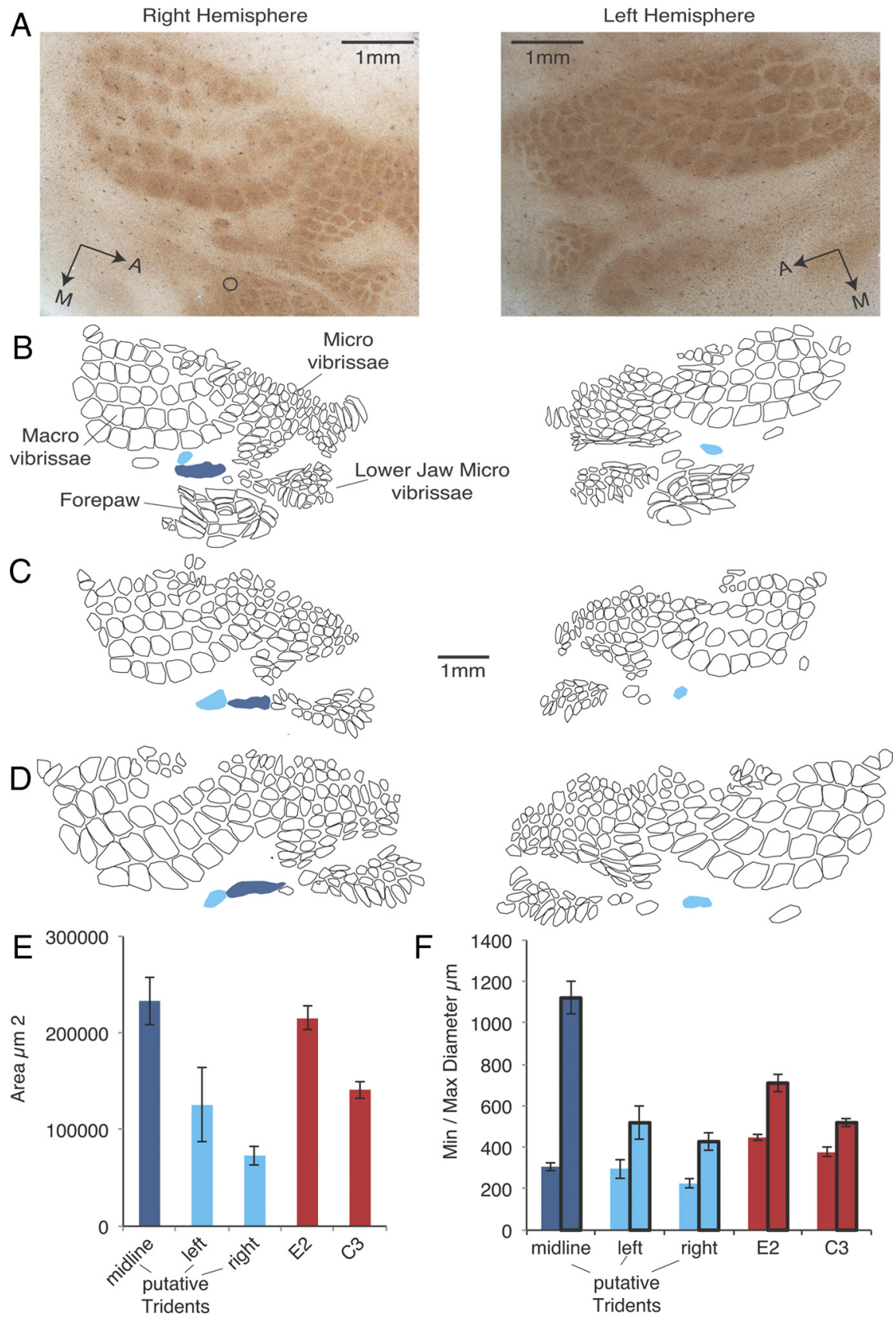

Figure 5. Identification of putative trident barrels in flattened barrel cortices. $A$, Micrographs of flattened right and left somatosensory cortices of a rat histochemically stained for cytochrome-oxidase activity. Dark brown indicates zones of heightened (metabolic) cytochrome-oxidase activity, thus revealing whisker and paw barrels. Sections showing the putative trident barrel most clearly were chosen (highlighted in color in the drawings below); nevertheless these barrels are less clearly defined than the adjacent facial barrels. $\boldsymbol{B}$, Camera lucida drawing of shapes of whisker and forepaw barrels from the brain shown in $\boldsymbol{A}$. Note that the drawing is based on several serial sections (in addition to the one shown above) and is shown at a reduced scale. The drawing is slightly rotated such that the anterior-posterior body axis runs horizontally. The putative trident barrels are shown as filled shapes in light blue (left and right trident) and dark blue (midline trident). Putative trident barrels were uniquely identified on the basis of the following four criteria: (1) the coordinates estimated from physiology and the map of Chapin and Lin (1984), (2) extracellular mapping of receptive fields combined with histology, (3) their topographic location in the face representation, (4) the larger size of trident whiskers and the assumed larger size of trident barrels compared with the adjacent posterior lower jaw whisker barrels, and (5) their number $(n=3)$. C, D, Two further barrel drawings of pairs of left and right somatosensory cortices. These drawings are restricted to whisker barrels; paw barrels are not shown. Other conventions are as in $\boldsymbol{B}$. $\boldsymbol{E}$, Areas of trident barrels and the facial barrels E2 (the largest facial barrel) and C3 (an average size facial barrel). $\boldsymbol{F}$, Minimal and maximal (with black border) Feret diameter of trident and facial barrels. The Feret diameter is the shortest/longest border-to-border line crossing the shape's center. Data in $\boldsymbol{E}$ and $\boldsymbol{F}$ refer to flattened barrel cortices from 10 hemispheres of five animals resulting in five trident barrels each and $10 \mathrm{E} 2$, C 3 barrels from both hemispheres. Because cortices were flattened, absolute area and diameter estimates might be overestimates. Error bars indicate SEM.

\section{Physiological responses to trident whisker stimulation}

To test the neural representation of the submandibular-trident whiskers, we targeted whole-cell patch recordings to the putative trident barrels, as informed by the map of Chapin and Lin (1984). We recorded postsynaptic potentials as air puffs deflected the trident whiskers. In all cells recorded from the putative barrels, postsynaptic responses were observed. A biphasic response was typical (Fig. $6 A-C$, top row) with an initial EPSP preceding an IPSP. Responses lasted the duration of the stimulus.

In our recordings from left and right hemispheric trident representations, we obtained additional indications for a right hemispheric bias in the representation of the midline trident. In two cells recorded in the left hemisphere, we observed shorter latencies and strong postsynaptic responses to air puffs directed selectively to the right trident and long latencies and weak postsynaptic responses to air puffs directed selectively to the midline trident and the left trident whisker. In contrast, in a right hemispheric cell, we observed shorter latencies and strong responses for both the midline trident and left trident and long latencies and weak responses to the right trident. Our extracellular recording and receptive field mapping experiments further suggested that although the left and right trident whiskers were represented primarily contralaterally, there were bilateral responses to the midline trident whisker.

We hypothesized that the anatomical and biophysical differences between the submandibular trident whiskers and the facial whiskers would likely influence how they responded to similar stimulation. To test this hypothesis, we also targeted whole-cell patch recordings to the facial barrels. There, postsynaptic potentials were similarly biphasic (Fig. $6 A-C$, bottom row), although there were significant differences in the timing of each phase of the response. The rise time to the peak of the EPSP was slower in the submandibular trident whisker barrels than in the facial whisker barrels. Likewise, the absolute latency to the peak of the EPSP and IPSP was slower in the submandibulartrident than in the facial whisker barrels (Fig. 6E,F). However, the amplitude of each event was not significantly different (Fig. 6D). We conclude that facial and trident whiskers do not only differ in their morphology, behavioral use, and biomechanics, but also show remarkable differences in the response dynamics. 


\section{Discussion}

Here we provide a description of the rat submandibular whisker trident. First, we point out structural and functional differences between trident and facial whiskers. In the second part of the discussion, we outline a theory of the operation of trident whiskers. Accordingly, we propose an idiothetic hypothesis in which the trident whiskers function as a tactile speedometer and estimate heading direction.

Unlike facial whiskers, tridents do not function in high-resolution object exploration

A key result of our investigation is that trident whiskers are functionally very different from facial whiskers. Specifically, we suggest that trident whiskers do not operate for object exploration as facial whiskers do. This conclusion rests on the following observations. First, the small number of trident whiskers and the relatively wide spacing of their tips prevented them from signaling fine-grained tactile information (Fig. 1A). Second, the physiology showing poor temporal resolution of trident responses suggests that these whiskers do not provide temporally precise, high-resolution signals to the animal. Third, the nonexposed body position trident whiskers behind the face/ below the chin is a unique feature of the trident system not shared by other whiskers (Fig. 1B). Fourth, trident whiskers are typically not touching anything and do not engage in active touch and object exploration (Fig. 2). Finally, the trident whisker barrels are isolated from each other and other barrels. They are elongated and discontinuous in size. This is in stark contrast to the compact and continuous representation of facial whiskers. In contrast, the shape, size, and packing of facial whisker barrels allow a compact representation of facial whiskers. Facial whisker barrels and barrels of other whiskers are represented close together and the Dirichlet domain shape of facial whisker barrels allows for a maximally compact whisker representation (Senft and Woolsey, 1991). The size of facial whisker barrels changes gradually from barrel to barrel.

\section{The trident whiskers as idiothetic ego-motion sensors and tactile speedometer}

Idiothetic sensing

The functional differences between trident and facial whiskers and the lack of conventional allothetic object or obstacle trident contacts are at first puzzling to the sensory physiologist. However, a strong clue to the function of trident whiskers comes from our behavioral analysis. Our observations demonstrated that trident whiskers engage in contact during only very specific behavioral circumstances. In particular, we found that trident whiskers engaged in ground contact during specific kinds of locomotion, namely during headdown running in novel environments or in known environments during foraging. Other types of locomotion, such as roaming in a home cage, do not involve head-down running or trident-ground contacts. During exploration of a novel environment and foraging, the animal does not have a predetermined path; therefore, the trident is engaged when the animal needs to keep track of its path (i.e.,
B

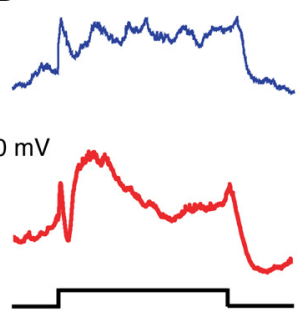

C
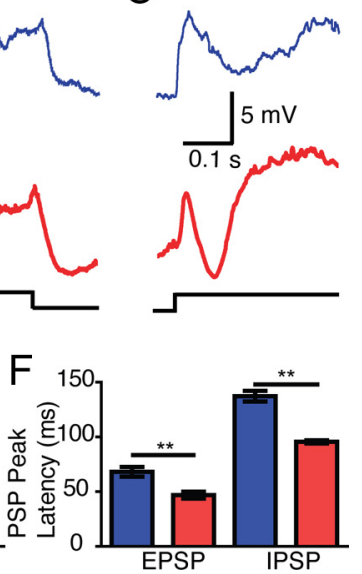

Figure 6. Postsynaptic responses to air puffs in the trident and facial whisker representations. Whole-cell recordings from the submandibular trident (blue) and facial (red) whisker barrels. All 11 of 11 cells from the putative submandibular-trident barrel responded to submandibular-trident air puff; 5 of 5 cells from the facial whisker barrel responded to the facial air puff. $\boldsymbol{A}$, Repreinse to air puff. $\boldsymbol{B}$, Average response from 10 trials in 1 cell. $\boldsymbol{C}$, Initial part of the response shown in $\boldsymbol{B}$ at a higher time using high-speed videography (our unpublished observations). Group data in $\boldsymbol{D}-\boldsymbol{F}$ indicate mean $\pm \mathrm{SE}$. Two submandibulartrident cells lacked an unambiguous initial EPSP phase and were not included in the group data. ( $t$ test, ${ }^{*} p<0.05 ;{ }^{* *} p<0.01$ ).

to perform path integration). We suggest that the trident system is uniquely suited for ego-motion sensing.

\section{Ground following and specializations of trident whiskers for continuous sensing}

The biomechanical data collected here show that trident whiskers have the capacity to follow caudal (corresponding to animalforward) ground movement very smoothly. Rostral movements of the ground lead to very jerky movements. This difference seems to result from a stable balance between frictional forces from ground movement and the force of the ground actively deflecting the trident. Rostral movements charge up the backwards-curved whisker like a spring, resulting in abrupt discharges of the spring force and jerky movements. Smooth ground following is a property that differentiates tridents from facial whiskers. Although the significance of smooth ground following is not yet clear, we suggest that it improves the ability of trident whiskers to derive continuous measurements about ground movement, such as heading direction and velocity (see our discussion below). The smoothness of whisker deflection will greatly reduce the variance of "continuous measurements" derived from ground contact. In contrast, facial whiskers do not engage in sustained ground contacts and might be specialized for transient object contact mediated by whisking. Our interpretation of smooth ground following as a specialization for continuous ground sensing is supported by our physiology demonstrating relatively poor temporal fidelity in trident cell responses and might also explain why facial whiskers do not show this property.

\section{Velocity sensitivity}

In our biomechanical experiments, trident angular displacement changed as a function of ground velocity. Such changes in deflection angle greatly exceed the angular resolution determined in psychophysical experiments involving facial whiskers (Stüttgen and Schwarz, 2008). Surprisingly, the midline trident whisker was deflected less as ground speed increased, pointing to changes in the contribution of static over dynamic friction in the trident-ground 
interaction. These experiments suggest that the trident whisker deflection angle could in principle be read out as a tactile speedometer.

\section{Midline trident might offer distinct advantages for heading direction estimates}

One of greatest riddles in our dataset relate to the midline trident whisker. Why is this whisker the rat's only unpaired whisker of several hundred whiskers? Why is the putative trident representation the animal's largest barrel whereas the midline trident whisker is infrequently in contact with an object and is a relatively small whisker in a secluded part of the body? We argue that the midline position of the midline trident offers unique advantages for heading direction estimates. Specifically, we suggest that the midline position might allow a particularly simple readout of heading direction, because the forces acting on a whisker in the midline position will be symmetric to ego-motion direction. When an animal turns right or left, a midline whisker will experience exactly the same mirror-symmetric forces, which will not be true for any non-midline whisker position. Therefore, we hypothesize that the midline trident acts as a kind of inverse "joystick" telling the animal where it is heading.

\section{Trident representation and the rat's idiothetic system}

Rats are thought to have powerful idiothetic capacities. Blind rats can build up robust spatial representations (place fields) in the absence of visual cues (Save et al., 1998). The role of the vestibular system in homing and path integration was demonstrated early on (Mittelstaedt and Mittelstaedt, 1980), and the discovery of headdirection cells (Taube et al., 1990) piqued interest in vestibular mechanisms involved in path integration. The available evidence indicates that trident whisker inputs are not the major driving force of head-direction signal, because head-direction cells in many parts of the brain are under the control of visual landmarks, depend on vestibular inputs, and are active when the animal is standing still (Taube, 2007). Even if trident signals are not the major driving force of head-direction cells, trident signals are well suited to interact synergistically with the vestibular system, and their anatomy suggests that such interactions occur. In head-down running, the trident whiskers are positioned exactly in the middle below the semicircular canals and, by virtue of midline body position, the trident signals come in the same head-centered format that is used by the headdirection signal. Most interestingly, the putative trident whisker barrels appear to strongly project to the rat's vestibular nuclei (Nishiike et al., 2000). Only four cortical areas appear to project to the spinal vestibular nucleus. The two major corticofugal inputs arise from the secondary somatosensory cortex and an area referred to as the lateral forelimb region (Nishiike et al., 2000). This so-called lateral forelimb region appears to be identical to the putative trident representation identified here. In addition, the corticocortical connections of the putative trident barrels appear to be consistent with a role of the trident system in path integration. The putative trident representation appears to be heavily connected to the rat posterior parietal cortex (Reep et al., 1994) and lesions to this area induce pathintegration deficits (Parron and Save, 2004). The projection from putative trident barrels to posterior parietal cortex localizes to the unresponsive zone lateral from the paw representation and, at least in some cases, appears to originate preferentially from the right hemisphere. What is remarkable about both the putative trident projection to the posterior parietal cortex and to the spinal vestibular nucleus is that these projections originate selectively from the putative trident system and do not involve the nearby facial whisker representation. Therefore, these connectivity patterns strongly suggest differential roles of trident and facial whiskers with respect to vestibular or path-integration functions.

\section{Conclusion}

We have described a novel whisker array of the rat, the submandibular whisker trident. These whiskers are unique in their morphology, behavioral engagement, biomechanics, and cortical representation. We have suggested that numerous idiosyncrasies of the trident system can be explained by an idiothetic function of these whiskers. This hypothesis predicts that neurons in the trident system will be sensitive to and will systematically represent ego-motion parameters such as heading direction and speed.

\section{References}

Anjum F, Turni H, Mulder PG, van der Burg J, Brecht M (2006) Tactile guidance of prey capture in Etruscan shrews. Proc Natl Acad Sci U S A 103:16544-16549. CrossRef Medline

Arabzadeh E, Zorzin E, Diamond ME (2005) Neuronal encoding of texture in the whisker sensory pathway. PLoS Biol 3:e17. CrossRef Medline

Brecht M (2007) Barrel cortex and whisker-mediated behaviors. Curr Opin Neurobiol 17:408-416. CrossRef Medline

Brecht M, Sakmann B (2002) Dynamic representation of whisker deflection by postsynaptic potentials in morphologically reconstructed spiny stellate and pyramidal cells in the barrels and septa of layer 4 in rat somatosensory cortex. J Physiol 543:49-70. CrossRef Medline

Brecht M, Preilowski B, Merzenich MM (1997) Functional architecture of the mystacial vibrissae. Behav Brain Res 84:81-97. CrossRef Medline

Brecht M, Roth A, Sakmann B (2003) Dynamic receptive fields of reconstructed pyramidal cells in layers 3 and 2 of rat somatosensory cortex. J Physiol 553:243-265. CrossRef Medline

Carvell GE, Simons DJ (1990) Biometric analyses of vibrissal tactile discrimination in the rat. J Neurosci 10:2638-2648. Medline

Chapin JK, Lin CS (1984) Mapping the body representation in the SI cortex of anesthetized and awake rats. J Comp Neurol 229:199-213. CrossRef Medline

Crochet S, Petersen CC (2006) Correlating whisker behavior with membrane potential in barrel cortex of awake mice. Nat Neurosci 9:608-610. CrossRef Medline

Derdikman D, Yu C, Haidarliu S, Bagdasarian K, Arieli A, Ahissar E (2006) Layer-specific touch-dependent facilitation and depression in the somatosensory cortex during active whisking. J Neurosci 26:9538-9547. CrossRef Medline

Deschênes M, Moore J, Kleinfeld D (2012) Sniffing and whisking in rodents. Curr Opin Neurobiol 22:243-250. CrossRef Medline

Elston GN, Pow DV, Calford MB (1997) Neuronal composition and morphology in layer IV of two vibrissal barrel subfields of rat cortex. Cereb Cortex 7:422-431. CrossRef Medline

Fee MS, Mitra PP, Kleinfeld D (1997) Central versus peripheral determinants of patterned spike activity in rat vibrissa cortex during whisking. J Neurophysiol 78:1144-1149. Medline

Finnerty GT, Roberts LS, Connors BW (1999) Sensory experience modifies the short-term dynamics of neocortical synapses. Nature 400:367-371. CrossRef Medline

Hartmann MJ, Johnson NJ, Towal RB, Assad C (2003) Mechanical characteristics of rat vibrissae: resonant frequencies and damping in isolated whiskers and in the awake behaving animal. J Neurosci 23:6510-6519. Medline

Jadhav SP, Feldman DE (2010) Texture coding in the whisker system. Curr Opin Neurobiol 20:313-318. CrossRef Medline

Manns ID, Sakmann B, Brecht M (2004) Sub- and suprathreshold receptive field properties of pyramidal neurons in layers $5 \mathrm{~A}$ and $5 \mathrm{~B}$ of rat somatosensory barrel cortex. J Physiol 556:601-622. CrossRef Medline

Margrie TW, Brecht M, Sakmann B (2002) In vivo low resistance whole-cell recordings from neurons in the anaesthetized and awake mammalian brain. Pflügers Arch 444:491-498. CrossRef Medline

Mittelstaedt ML, Mittelstaedt H (1980) Homing by path integration in a mammal. Naturwissenschaften 67:566-567. CrossRef

Moore CI, Nelson SB (1998) Spatio-temporal subthreshold receptive fields in the vibrissa representation of rat primary somatosensory cortex. J Neurophysiol 80:2882-2892. Medline

Neimark MA, Andermann ML, Hopfield JJ, Moore CI (2003) Vibrissa resonance as a transduction mechanism for tactile encoding. J Neurosci 23:6499-6509. Medline

Nishiike S, Guldin WO, Bäurle J (2000) Corticofugal connections between the 
cerebral cortex and the vestibular nuclei in the rat. J Comp Neurol 420:363-372. CrossRef Medline

O'Connor DH, Huber D, Svoboda K (2009) Reverse engineering the mouse brain. Nature 461:923-929. CrossRef Medline

Parron C, Save E (2004) Evidence for entorhinal and parietal cortices involvement in path-integration. Exp Brain Res 159:349-359. CrossRef Medline

Reep RL, Chandler HC, King V, Corwin JV (1994) Rat posterior parietal cortex: topography of corticocortical and thalamic connections. Exp Brain Res 100:67-84. CrossRef Medline

Ritt JT, Andermann ML, Moore CI (2008) Embodied information processing: vibrissa mechanics and texture features shape micromotions in actively sensing rats. Neuron 57:599-613. CrossRef Medline

Save E, Cressant A, Thinus-Blanc C, Poucet B (1998) Spatial firing of hippocampal place cells in blind rats. J Neurosci 18:1818-1826. Medline

Scanziani M, Häusser M (2009) Electrophysiology in the age of light. Nature 461:930-939. CrossRef Medline

Senft S L, Woolsey TA (1991) Mouse barrel cortex viewed as Dirichlet domains Cereb Cortex 1:348-363. CrossRef

Simons DJ (1978) Response properties of vibrissa units in rat SI somatosensory neocortex. J Neurophysiol 41:798-820. Medline

Simons DJ (1983) Multi-whisker stimulation and its effects on vibrissa units in rat SmI barrel cortex. Brain Res 276:178-182. CrossRef Medline

Simons DJ (1985) Temporal and spatial integration in the rat SI vibrissa cortex. J Neurophysiol 54:615-635. Medline
Stüttgen MC, Schwarz C (2008) Psychophysical and neurometric detection performance under stimulus uncertainty. Nat Neurosci 11:1091-1099. CrossRef Medline

Taube JS (2007) The head direction signal: origins and sensory-motor integration. Annu Rev Neurosci 30:181-207. CrossRef Medline

Taube JS, Muller RU, Ranck JB Jr (1990) Head-direction cells recorded from the postsubiculum in freely moving rats. I. Description and quantitative analysis. J Neurosci 10:420-435. Medline

Van der Loos H, Woolsey TA (1973) Somatosensory cortex: structural alterations following early injury to sense organs. Science 179:395-398. CrossRef Medline

von Heimendahl M, Itskov PM, Arabzadeh E, Diamond ME (2007) Neuronal activity in rat barrel cortex underlying texture discrimination. PLoS Biol 5:e305. CrossRef Medline

Wong-Riley M (1979) Changes in the visual system of monocularly sutured or enucleated cats demonstratable with cytochrome oxidase. Brain Res 171:11-28. CrossRef Medline

Woolsey TA, Van der Loos H (1970) The structural organization of layer IV in the somatosensory region (SI) of mouse cerebral cortex. The description of a cortical field composed of discrete cytoarchitectonic units. Brain Res 17:205-242. CrossRef Medline

Zhu JJ, Connors BW (1999) Intrinsic firing patterns and whisker-evoked synaptic responses of neurons in the rat barrel cortex. J Neurophysiol 81:1171-1183. Medline 